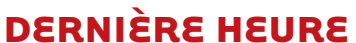

\title{
Identification d'une nouvelle cible des diurétiques thiazidiques dans le rein
}

Dominique Eladari, Régine Chambrey, Françoise Leviel

\section{Les transporteurs rénaux}

de sodium, déterminants majeurs

\section{de la pression artérielle}

La quantité de chlorure de sodium $(\mathrm{NaCl})$ contenue dans l'organisme est le principal facteur qui détermine le volume du fluide extracellulaire et, par conséquent, la pression artérielle. Ainsi, une rétention de sodium augmente la volémie, ce qui élève la pression artérielle, alors qu'une perte de sodium a pour conséquence les phénomènes inverses. La quantité de sodium est maintenue à un niveau très stable malgré des apports alimentaires pouvant varier considérablement au cours du temps et d'un individu à l'autre. Pour assurer cette stabilité, le rein filtre chaque jour une grande quantité de sodium au niveau du filtre glomérulaire (environ 25 moles, ce qui correspond à environ 1,5 kg de sel de table) dont 99,6\% seront réabsorbés par les cellules épithéliales bordant la lumière du néphron. La fraction minime restante (environ 0,4\%) correspond à la millimole près à la quantité apportée par l'alimentation. Ainsi, la quantité de sodium présente dans l'organisme reste constante grâce à cet équilibre entre les apports alimentaires et l'excrétion urinaire. Les mécanismes à l'origine de l'hypertension artérielle restent largement inconnus. Néanmoins, ils semblent apparaître avec l'âge et être largement favorisés par un régime riche en sel, avec une tendance du rein à ne plus être capable d'équilibrer parfaitement les entrées digestives et les sorties urinaires de sodium qui deviennent insuffisantes. Ceci provoque l'accumulation progressive de sodium dans l'organisme, une augmentation de la volémie et finalement une hypertension artérielle. Par ailleurs, les aberrations de la régulation du transport de $\mathrm{NaCl}$, en particulier dans la partie terminale du néphron (tubule contourné distal, tube connecteur et canal collecteur), conduisent à une rétention excessive de sodium dans l'organisme qui est à l'origine des syndromes œdémateux. Par conséquent, les médecins ont depuis longtemps perçu l'intérêt thérapeutique majeur des composés pouvant augmenter l'excrétion rénale du sodium: les diurétiques.

La réabsorption rénale du sodium a lieu tout le long du néphron et est effectuée par des protéines spécialisées, les transporteurs de sodium, exprimées au pôle apical des cellules qui capturent le sodium de l'urine primitive pour le faire entrer dans les cellules, et la pompe à sodium basolatérale, la $\mathrm{Na}^{+} / \mathrm{K}^{+}$-ATPase, qui fait sortir le sodium de la cellule vers le sang au prix d'une consommation d'énergie. Les diurétiques, qui sont en fait des agents natriurétiques (qui augmentent l'élimination urinaire de sodium), agissent en inhibant les transporteurs, et l'identification précise de leurs cibles moléculaires respectives a été à l'origine de la connaissance de la physiologie moléculaire des transporteurs épithéliaux. Cette identification a été initialement basée sur des observations physiologiques chez l'animal.
D. Eladari, F. Leviel :

UPMC Université Paris-6,

Inserm UMRS 872 et CNRS ERL7226,

Laboratoire de génomique,

physiologie et physiopathologie

rénales, Centre de recherche des Cordeliers,

équipe 3 , Escalier $\varepsilon-R D C$,

15, rue de l'École de médecine,

75006 Paris, France;

Faculté de médecine Paris Descartes,

Université Paris Descartes, Paris ;

département de physiologie,

Groupe Necker Enfants Malades-HEGP, APHP,

Paris, France.

R. Chambrey : UPMC Université Paris-6,

Inserm UMRS 872 et CNRS ERL7226,

Laboratoire de génomique,

physiologie et physiopathologie rénales,

Centre de recherche des Cordeliers,

Paris, France.

dominique.eladari@crc.jussieu.fr

Le site d'action des différentes classes de diurétiques a été d'abord identifié grâce à des études de clairance du sodium, du chlore et du potassium, et grâce au développement de microtechniques, comme la microponction de segments du néphron in vivo ou la microperfusion in vitro de segments de néphron isolé. Par la suite, la confirmation des transporteurs impliqués est devenue possible grâce aux techniques de clonage et d'expression hétérologue et aux études génétiques ayant permis d'élucider l'origine des syndromes humains qui reproduisent les effets de l'administration des différentes classes de diurétiques. Ainsi, quatre transporteurs de sodium jouant un rôle majeur dans la régulation de la balance sodée on été distingués (pour revue voir [1]). II s'agit de l'échangeur $\mathrm{Na}^{+} / \mathrm{H}^{+} \mathrm{NHE3}$ $\left(\mathrm{Na}^{+} / \mathrm{H}^{+}\right.$exchanger isoform 3$)$ qui est la cible de l'acétazolamide et qui est présent dans le tube contourné proximal, le cotransporteur NKCC2 $\left(\mathrm{Na}^{+}-\mathrm{K}^{+}-2 \mathrm{Cl}^{-} \mathrm{CO}-\right.$ transporter 2) inhibé par le furosémide et qui réabsorbe le sodium dans l'anse de Henlé, le cotransporteur NCC $\left(\mathrm{Na}^{+}-\mathrm{Cl}^{-}\right.$ 

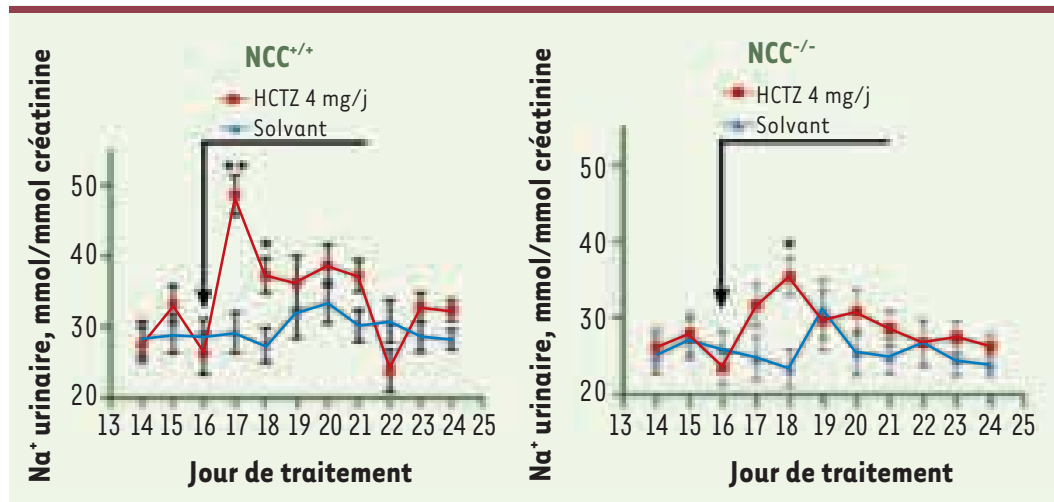

cotransporter) inhibé par les diurétiques thiazidiques dans le tube contourné distal, et enfin le canal à sodium $\mathrm{ENaC}$ (epithelial $\mathrm{Na}^{+}$channel) des cellules principales du canal collecteur, sensible à l'amiloride. Des mutations dans I'un ou l'autre de ces transporteurs sont impliquées dans des désordres génétiques rares modifiant la pression artérielle. En outre, il a été démontré récemment que des modifications partielles de l'activité de certaines de ces molécules influencent le niveau de pression artérielle au niveau de la population [2].

\section{L'efficacité des diurétiques}

thiazidiques dans le traitement de l'hypertension reste mystérieuse Parmi les différentes classes de diurétiques inscrites à la pharmacopée, la classe des diurétiques thiazidiques occupe une place tout à fait primordiale. L'hydrochlorothiazide est l'un des plus anciens agents diurétiques identifiés. Ce composé, initialement développé par modification chimique de l'acétazolamide, un autre diurétique [3], et ses dérivés, figurent en 2010 parmi les diurétiques les plus prescrits dans le monde. En effet, les thiazidiques font partie des agents recommandés en première intention dans le traitement de l'hypertension artérielle essentielle non compliquée. La grande majorité des études qui visent à traiter l'hypertension incluent systématiquement un diurétique thiazidique en première intention ou en association avec d'autres classes d'antihypertenseurs. L'importance de ces diurétiques est tout simplement due au fait qu'ils font partie des rares molécules à avoir démontré leur efficacité tant sur le contrôle des chiffres tensionnels que sur la diminution de la mortalité ou des morbidités associées à l'élévation de la pression artérielle [4, 5, 10]. Cette efficacité est cependant surprenante. $\varepsilon n$ effet, la cible classique des diurétiques thiazidiques, le cotransporteur NCC, est exprimée dans un segment du néphron, le tube contourné distal, qui représente moins de $5 \%$ de la masse épithéliale rénale et qui réabsorbe seulement $7 \%$ de la quantité de sodium filtrée par le glomérule. Par comparaison, le cotransporteur NKCC2, qui est la cible d'un autre diurétique, le furosémide, est responsable de la réabsorption de $30 \%$ de cette charge filtrée. En d'autres termes, bien qu'il ait une action diurétique quatre fois moins puissante que le furosémide, I'hydrochlorothiazide semble bien plus efficace. De plus, certains effets bénéfiques ou néfastes de l'hydrochlorothiazide sont difficilement expliqués. Par exemple, l'hydrochlorothiazide favorise la survenue d'un syndrome métabolique ou favorise la vasodilatation artérielle, alors que ni les artères, ni le pancréas, le muscle, le foie ou les adipocytes n'expriment NCC.

\section{Un nouveau système}

\section{de transport de sodium identifié}

dans le rein mais non restreint au rein Afin d'expliquer ces mécanismes d'action de l'hydrochlorothiazide, nous avons tout d'abord voulu démontrer de
Figure 1. Évolution de la natriurèse après administration de $4 \mathrm{mg}$ par jour d'hydrochlorothiazide (HCTZ) ou du solvant chez des souris $\mathrm{NCC}^{+/+}$ou $\mathrm{NCC}^{-/-}$. Chez les souris $\mathrm{NCC}^{+/+}$, la natriurèse est stimulée dès le jour Jl de l'administration et reste supérieure, à J2, à celle des souris auxquelles on administre le solvant. Chez les souris $\mathrm{NCC}^{-/-}$, bien que la réponse soit moindre en amplitude, la natriurèse est stimulée par l'HCTZ au deuxième jour attestant de la présence d'une autre cible moléculaire que NCC pour l'HCTZ.

façon indiscutable que tous les effets de ce composé ne sont pas liés à l'inhibition du cotransporteur NCC. Nous suspections l'existence d'un autre transporteur de sodium sensible aux thiazidiques dans les cellules épithéliales rénales car des études antérieures sur des canaux collecteurs de rat isolés et perfusés in vitro avaient démontré que ce diurétique est capable d'inhiber $50 \%$ de l'absorption de sodium alors que ce segment de néphron n'exprime pas NCC. Nous avons donc dans un premier temps administré le diurétique à des souris KO (knock-out) pour l'expression de NCC [6]. En effet, en l'absence de la cible moléculaire de l'hydrochlorothiazide, la réponse à ce composé aurait dû être nulle chez ces souris. Pourtant, l'hydrochlorothiazide est capable de bloquer une fraction de l'absorption de sodium (Figure 1) et donc d'augmenter la natriurèse des souris, et ceci malgré l'absence du cotransporteur NCC, ce qui prouve la présence d'un autre transporteur de sodium sensible à ce composé. Compte tenu de l'étude antérieure qui suggère que ce transporteur est localisé dans le canal collecteur, nous avons ensuite caractérisé le transport de sodium et de chlore dans des canaux collecteurs isolés manuellement par microdissection et perfusés in vitro. $\varepsilon n$ couplant l'usage de différents diurétiques à l'utilisation de souris KO pour différents transporteurs de sodium nous avons démontré de façon directe que le canal collecteur exprime deux systèmes de transport de sodium [7] : (1) le canal 


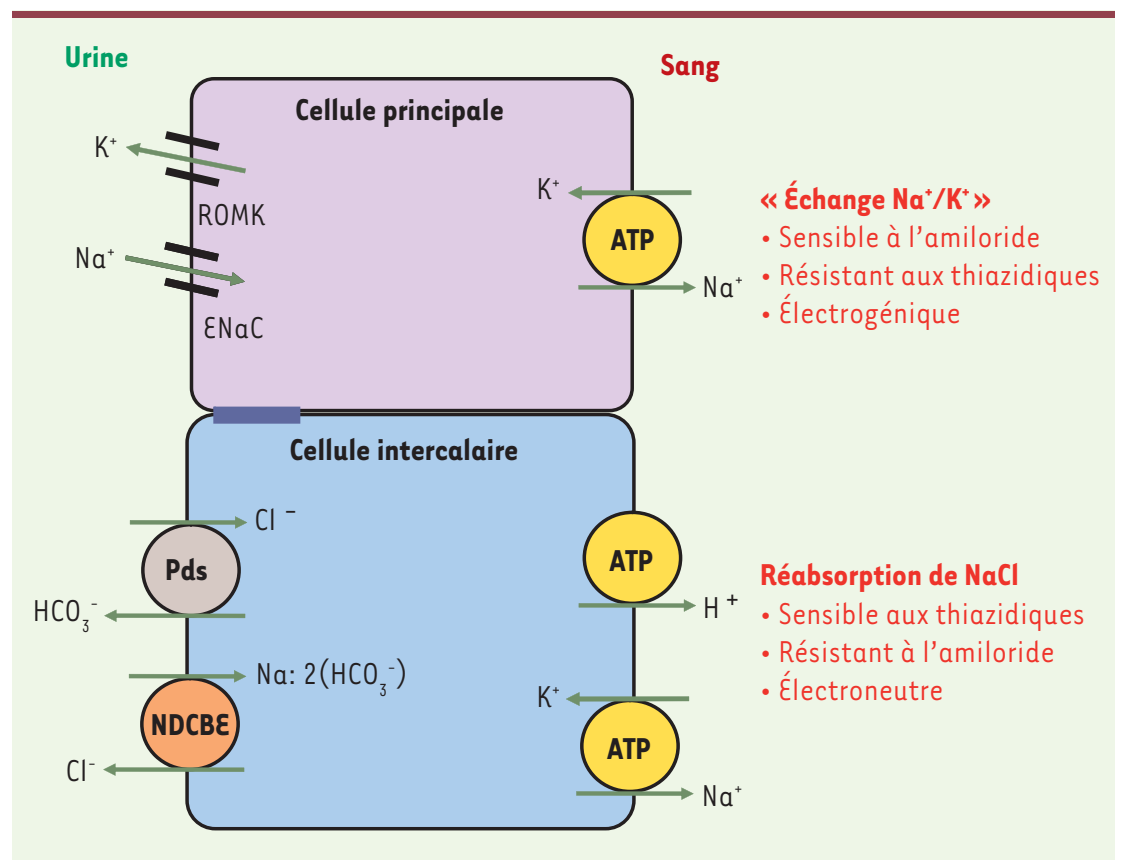

à sodium épithélial $\varepsilon \mathrm{NaC}$ qui représente le système «classique » et dont la caractéristique est de relayer un transport électrogénique de sodium, favorisant la sécrétion de potassium, insensible à l'hydrochlorothiazide et inhibé par l'amiloride ; (2) un transport de $\mathrm{NaCl}$ électroneutre, sensible à I'hydrochlorothiazide et insensible à l'amiloride et qui résulte du couplage fonctionnel de deux échangeurs d'anions: la pendrine et NDCBE (pour $\mathrm{Na}^{+}$-driven chloride bicarbonate exchanger) (Figure 2).

Par ailleurs, nous avons démontré que l'expression de $N D C B E$, contrairement à NCC, n'est pas restreinte au rein, ce qui pourrait expliquer certains des effets des diurétiques thiazidiques, en particulier au niveau des artères et du cerveau.

Les cellules intercalaires, de nouveaux participants

à la régulation de la pression artérielle Un nouvel aspect de notre travail est l'identification de la localisation de ce nouveau système de transport de $\mathrm{NaCl}$ dans les cellules intercalaires du canal collecteur. En effet, le canal collecteur représente le dernier segment de néphron avant l'excrétion de l'urine dans les voies urinaires. Cet épithélium a donc
Figure 2. Schéma des principaux systèmes de transport impliqués dans l'absorption de sodium dans le canal collecteur. $\mathrm{ENaC}$ : epithelial sodium channel; ROMK: rectifying outer medullary $\mathrm{K}^{+}$channel ; Pds : pendrine ; NDCBE : $\mathrm{Na}$-driven $\mathrm{Cl}^{-}$bicarbonate exchanger.

intercalaires de type $B$, qui expriment la pompe à protons au pôle basolatéral et l'échangeur $\mathrm{Cl}^{-} / \mathrm{HCO}_{3}{ }^{-}$pendrine au pôle urinaire, servent à excréter des bases et à absorber de l'acide.

Nous démontrons dans notre étude que le même échangeur apical pendrine des cellules intercalaires de type $B$ peut également fonctionner couplé à NDCBE pour réaliser non plus un échange $\mathrm{Cl}^{-}$ $/ \mathrm{HCO}_{3}{ }^{-}$, mais bien une absorption nette de $\mathrm{NaCl}$.

Nos données impliquent donc qu'il existe en fait une intrication plus poussée des différentes fonctions homéostatiques de l'épithélium du canal collecteur et suggèrent que le schéma classique dans lequel les cellules principales régulent la volémie et la pression artérielle, alors que les cellules intercalaires servent exclusivement à réguler l'état acidebase, doit être remis à jour. sition cellulaire hétérogène. Se distinguent ainsi les cellules principales et les cellules intercalaires, elles-mêmes subdivisées en deux types $A$ et $B$, sur la base de la polarisation de la $\mathrm{H}^{+}$-ATPase vacuolaire. Classiquement, chacun de ces types cellulaires différents a une fonction extrêmement définie. Ainsi, les cellules principales qui sont équipées d'aquaporines (canaux à eau), du canal épithélial à sodium $\varepsilon \mathrm{NaC}$ et du canal potassique ROMK (rectifying outer medullary potassium channel) sont considérées comme dédiées à la réabsorption de sodium et d'eau et à l'élimination du potassium. Les cellules intercalaires de type A expriment la pompe à protons vers le pôle urinaire (apical) et l'échangeur $\mathrm{Cl}^{-} / \mathrm{HCO}_{3}^{-} \mathrm{A} \varepsilon \mathrm{l}$ (anion exchanger, aussi connu sous le nom de bande 3) au pôle basolatéral et sont donc équipées pour excréter de l'acide et absorber du bicarbonate. Les cellules

\section{La physiologie, une discipline} toujours d'actualité pour développer des stratégies thérapeutiques ciblées Au-delà de l'identification d'un nouveau transporteur de sodium et d'une nouvelle cible des diurétiques thiazidiques dans le rein, l'observation que nous rapportons illustre parfaitement une des nouvelles tendances en physiologie. $\varepsilon n$ effet, l'identification de NDCBE comme nouvelle cible des diurétiques thiazidiques a été la conséquence directe de I'utilisation des modèles de souris dont les gènes codant les différents transporteurs rénaux ont été spécifiquement inactivés. L'implication de ces transporteurs dans le transport transépithélial de sodium a été éliminée ou confirmée de façon bien plus directe et définitive qu'en utilisant des agents pharmacologiques. Les modèles de souris KO pour 
les différents transporteurs avaient déjà été générés et étudiés en détail par d'autres laboratoires. L'identification de la nouvelle cible de l'hydrochlorothiazide n'a été rendue possible que grâce à l'utilisation de techniques physiologiques anciennes que nous avons maintenues au laboratoire et adaptées à la souris, ce qui nous a permis d'étudier directement la fonction de transport transépithélial sur des tubules isolés du tissu frais. Cette technique de microperfusion de tubules isolés, qui a été développée dans les années 1960 [8], est progressivement tombée en désuétude comme la plupart des techniques physiologiques, et a quasiment disparu des laboratoires du monde entier depuis 1980. Nos résultats démontrent que ces méthodes restent particulièrement utiles et plaident pour une intégration de ces approches «anciennes » hautement spécialisées dans l'étude de la fonction de la molécule dans l'organisme ainsi que dans l'étude d'agents pharmacologiques potentiels. $\diamond$
Identification of a new target for thiazidic diuretics in kidney

\section{REMERCIEMENTS}

Ce travail est financé par l'Inserm, il bénéficie du financement ANR Physio 2007-RV07084 attribué à $D$. Eladari et est financé également par le réseau transatlantique pour la recherche sur l'hypertension (TNH) de la fondation Leducq.

\section{CONFLIT D'INTÉRÊTS}

Les auteurs déclarent n'avoir aucun conflit d'intérêts concernant les données publiées dans cet article.

\section{RéFÉRENCES}

1. Lifton RP, Gharavi AG, Geller DS. Molecular mechanisms of human hypertension. Cell 2001 ; $104: 545-56$.

2. Ji W, Foo JN, O'Roak BJ, et al. Rare independent mutations in renal salt handling genes contribute to blood pressure variation. Nat Genet 2008 ; $40: 592-9$.

3. Beyer KH Jr, Baer JE, Russo HF, et al. Electrolyte excretion as influenced by chlorothiazide. Science 1958 ; $127:$ 146-7.

4. Chobanian AV, Bakris GL, Black HR, et al. The seventh report of the joint national committee on prevention, detection, evaluation, and treatment of high blood pressure: the JNC 7 report. JAMA 2003; $289: 2560-72$.

5. ALLHAT officers and coordinators for the ALLHAT collaborative research group. Major outcomes in high-risk hypertensive patients randomized to angiotensin-converting enzyme inhibitor or calcium channel blocker vs diuretic: the antihypertensive and lipid-lowering treatment to prevent heart attack trial (ALLHAT). JAMA 2002; 288 : 2981-97.

6. Schultheis PJ, Lorenz JN, Meneton P, et al. Phenotype resembling Gitelman's syndrome in mice lacking the apical $\mathrm{Na}^{+}-\mathrm{Cl}^{-}$cotransporter of the distal convoluted tubule. J Biol Chem 1998 ; 273 : 29150-5.

7. Leviel F, Hübner CA, Houillier $\mathrm{P}$, et al. The $\mathrm{Na}^{+}-$ dependent chloride-bicarbonate exchanger SLC4A8 mediates an electroneutral $\mathrm{Na}^{+}$reabsorption process in the renal cortical collecting ducts of mice. J Clin Invest 2010; 12 avril (online).

8. Burg M, Grantham J, Abramow M, et al. Preparation and study of fragments of single rabbit nephrons. Am J Physiol 1966; 210 : 1293-8.

9. Hadchouel J, Delaloy C, Jeunemaitre X. WNKI et WNK4, nouveaux acteurs de l'homéostasie hydrosodée. Med Sci (Paris) $2005 ; 21: 55-60$.

10. Lanthier L, Nawar T, Plante GE. Résultats de l'étude ALLHAT : un traitement uniformisé de l'hypertension artérielle? Med Sci (Paris) 2003; $19: 377-80$.
TIRÉS À PART

D. Eladari

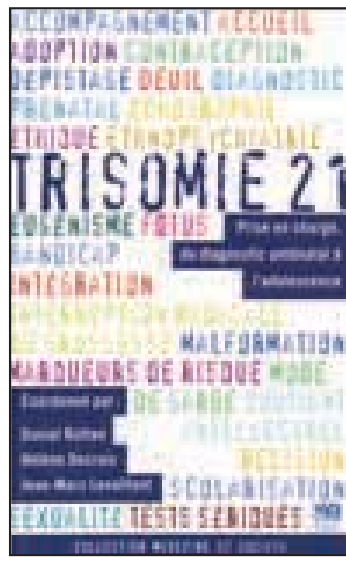

ISBN : 2-84254-105-7 248 pages

\section{Bon de commande}

À retourner à EDK, 2, rue Troyon - 92316 Sèvres Cedex

Tél. : 0155641393 - Fax : 0155641394 - E-mail : edk@edk.fr

NOM :

Prénom :

Adresse :

Code postal :

Ville :

Pays :

Fonction :

Je souhaite recevoir l'ouvrage Trisomie $21: 15 €+3 €$ de port $=\mathbf{1 8} €$ TTC

en $. . . \ldots \ldots . . .$. exemplaire, soit un total de $\ldots \ldots \ldots \ldots \ldots \ldots \ldots \ldots \ldots . . . . . \ldots$
$\square$ Par chèque, à l'ordre de $\mathbf{E} \mathbf{D} \mathbf{K}$
$\square$ Par carte bancaire : $\square$ Visa $\square$ Eurocard/Mastercard

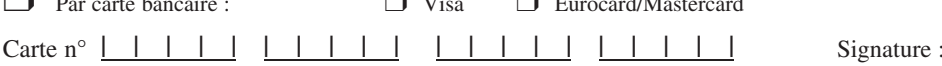

Date d'expiration: $\quad \underline{\mid}|\underline{1}|$

$\mathrm{N}^{\circ}$ de contrôle au dos de la carte : $\quad$ । $\quad$ । $\mid$ 
Médecine/Sciences est une revue internationale mensuelle francophone d'information dans tous les domaines de la biologie et de la médecine.

Les articles sont référencés dans PubMed depuis 2003, à l'exception des Brèves.

$\varepsilon n$ écrivant dans $\mathrm{m} / \mathrm{s}$, les auteurs ont le plaisir de faire partager aux lecteurs, dans leur langue, leur intérêt et même leur enthousiasme pour le sujet traité, en y apportant leur touche d'humour et de culture, surtout dans la partie Forum, où la plus grande liberté d'expression est autorisée.

Toutefois, la revue s'adressant à un lectorat très varié de scientifiques confirmés, d'enseignants, d'étudiants et de médecins, elle exige de la part des auteurs de développer leurs sujets, en allant jusqu'au bout des connaissances scientifiques, quelle qu'en soit la difficulté. Cela est particulièrement vrai pour les Synthèses, états de l'art sur un sujet donné, dans un style clair et intelligible pour les non-spécialistes.

La rédaction de la revue demande à tout auteur de se conformer aux quelques règles de base suivantes: - toujours définir les sigles et acronymes; $•$ rassembler en tableaux, glossaires, les précisions techniques, méthodologiques et les compléments d'information qui surchargeraient le texte.

\section{Les 3 rubriques de $m / s$}

1. Des Synthèses qui font le point sur un sujet par un (des) auteur(s) spécialiste(s) du domaine. Au-delà du catalogue des faits collectés, elles doivent permettre une vraie discussion des résultats scientifiques. Des Dossiers techniques (exposé d'une technique ou d'un ensemble de techniques récemment développées dans le domaine biomédical).

2. Le Forum propose des articles de réflexion, c'est-à-dire, des Perspectives et des Chroniques sur des sujets faisant l'objet de débats dans la communauté scientifique, ainsi que des revues sur l'histoire biomédicale, les sciences sociales et la santé, la santé et l'environnement... Les Faits et Chiffres traitent de sujets dans les champs de l'épidémiologie, de la démographie, de l'économie de la santé..., avec des données chiffrées sous forme de tableaux.

3. Le Magazine est le reflet de l'actualité scientifique, faisant état, dans des textes courts, de résultats originaux importants récemment publiés. II est constitué de Nouvelles, spontanées ou sollicitées, et de Brèves, courtes notes de lecture.

\section{Normes générales de présentation des articles}

Tous les articles doivent être soumis par voie électronique via le système Fontisworks (http://msc.fontismedia.com). La procédure est indiquée sur le site.

Attention : veiller à ce que les courriels provenant du site de gestion fontismedia ne soient pas reconnus comme spams par votre serveur et éliminés. Autoriser l'adresse msc. fontismedia.com

Tous les articles doivent être accompagnés des coordonnées de tous les auteurs : • nom et prénom, - institution, - adresse professionnelle, téléphone, télécopie, courriel.

Les Synthèses et les textes de la partie Forum ne peuvent excéder 18000 caractères (espaces compris, références exclues), 30 références et 3 à 5 illustrations (figures et tableaux), avec un titre en français et en anglais. Ils doivent être accompagnés d'un résumé d'environ 700 caractères destiné à offrir un aperçu rapide du sujet, et d'un résumé en anglais d'environ 1000 caractères, qui apparaîtra dans PubMed.

Il est demandé aux auteurs de mentionner les articles parus dans $\mathrm{m} / \mathrm{s}$ sur le sujet traité dans les dernières années (rechercher dans Pubmed).

\section{Les Nouvelles}

6000 à 8000 caractères au maximum (espaces compris, références exclues), 10 références au plus et 1 à 2 figures. Titre en français et en anglais.

\section{Les Brèves}

200 caractères au maximum (espaces compris), maximum de 5 références ne mentionnant que le premier auteur et les références du journal. Ex : Dupont T et al. Science $2010 ; 352: 458-9$.

Textes et tableaux en fichiers Word (enregistrements en .doc, format PDF exclus) - illustrations en fichiers séparés. Tableaux et illustrations appelés dans le texte. Les références citées dans les tableaux doivent apparaître sous forme de $\left[\mathrm{n}^{\circ}\right]$ et être incluses dans la liste des références.

Illustrations: schémas en format Illustrator ou PowerPoint, photos en format jpeg ou tif. Lorsque nécessaire, l'échelle de l'image doit figurer dans l'illustration et sa valeur indiquée dans la légende. Légendes complètes et détaillées des figures et tableaux intégrées en fin de texte. Illustrations en chiffres arabes (ex: Figure 1) et tableaux en chiffres romains (ex: Tableau II). Les illustrations doivent faire l'objet d'une demande d'autorisation de reproduction, si nécessaire.

\section{Présentation des références}

Appelées dans le texte par leurs numéros entre crochets ([1], [2], [3-5]) et classées par ordre d'apparition dans l'article.

Mentionner tous les noms des auteurs, suivis des initiales de leurs prénoms, jusqu'au nombre de 4 . Au-delà, les 3 premiers le sont, suivis de et al. (en italique).

Le style $M / S$ est inclus dans la liste des styles référencés dans EndNote (voir liste complémentaire «get more on the web » téléchargeable à partir de la rubrique style manager du site Endnote)

Pour les articles de revues scientifiques

Exemple: Sivori S, Falco M, Della Chiesa M, et al. CpG and double-stranded RNA trigger human NK cells by Toll-like receptors : induction of cytokine release and cytotoxicity against tumors and dendritic cells. Proc Natl Acad Sci USA 2004 ; 101 : 10116-21.

\section{Pour les ouvrages}

Exemple : Kupiec JJ, Sonigo P. Ni Dieu ni gène. Paris : Seuil, $2004: 230$ p.

\section{Pour les chapitres d'ouvrages}

Exemple : Ménard D, Beaulieu JF, Boudreau F, et al. Gastrointestinal tract. In : Unsicker K, Krieglstein K, eds. Cell signaling and growth factors. New York: Wiley, 2005 : 755-90.

Très important: les auteurs sont priés de mentionner tout conflit d'intérêt potentiel concernant le manuscrit soumis à publication dans $\mathrm{m} / \mathrm{s}$, en particulier de nature financière. Cette information sera gardée confidentielle par la rédaction de $\mathrm{m} / \mathrm{s}$ jusqu'à la publication de l'article.

\section{Rédaction Paris}

secretariat@medecinesciences.org

tél : 0155641393

Rédaction Québec :

medecine.sciences@bellnet.ca 


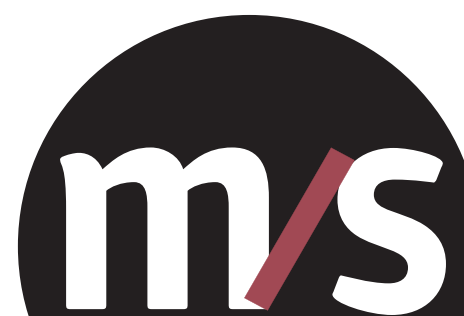

MEDECINE SCIENCES

Chaque mois,

avec les articles de référence de M/S

Chaque jour, sur www.medecinesciences.org

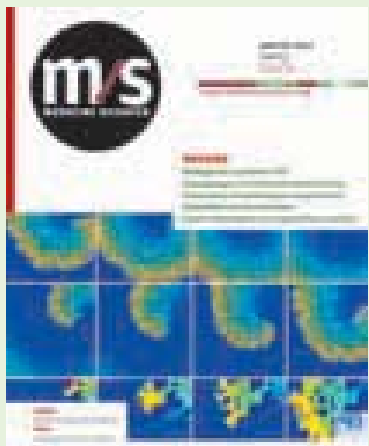

Médecine/Sciences est indexé dans

\section{PubMed/Medline}

Current Contents, série Life Sciences

EMBASE/Excerpta Medica

PASCAL

CABS

BIOSIS

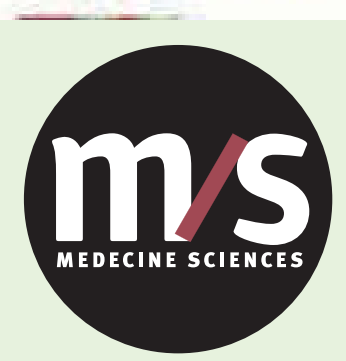

Tarifs d'abonnement M/S - 2010

Mensuel - 10 numéros/an

Abonnez-vous

\section{à Médecine/Sciences}

> Des articles rédigés par des médecins et des chercheurs reconnus sur la scène internationale qui posent avec rigueur les bases des débats scientifiques.

> Des synthèses, éditoriaux, dossiers techniques et analyses toujours replacés dans leur contexte pour que l'information soit la plus exacte, intelligible et objective.

> La dimension humaine privilégiée, avec l'analyse des retombées diagnostiques, thérapeutiques, la prévention et l'éthique liées aux nouvelles avancées.

> Un panorama clair et concis de l'actualité scientifique: des nouvelles, des brèves, des données chiffrées, des repères et perspectives pour qu'aucun fait significatif ne vous échappe.
Je souhaite m'abonner à $M / S$ :

Nom :

Adresse

Code postal

Pays :

$\varepsilon$-mail-obligatoire
Prénom :

Ville :

\section{Mon règlement :}

$\square$ Par mail edk@edk.fr

Uniquement pour les paiements par carte bancaire

$\square$ Par fax en envoyant ce bulletin au 0155641394

Uniquement pour les paiements par carte bancaire

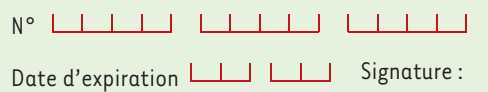

$N^{\circ}$ de contrôle au dos de la carte

$\square$ Par chèque à l'ordre de Médecine/Sciences, en envoyant ce bulletin à :

Éditions EDK

2, rue Troyon

92316 Sèvres Cedex, France

Pour recevoir une facture, cochez cette case $\square$
Je choisis l'abonnement:

\begin{tabular}{cc}
\multicolumn{2}{c}{ Particuliers } \\
Papier & Électronique \\
+ & seul \\
Électronique &
\end{tabular}

France

$\square 185 €$

$\square 125 €$

$U \varepsilon+$ autres

$\square 245 €$

$\square 125 €$

$$
\begin{array}{cc}
\multicolumn{2}{c}{\text { Institutions }} \\
\text { Papier } & \text { Électronique } \\
+ & \text { seul } \\
\text { Électronique } &
\end{array}
$$

$\square 415 € \quad \square$ Sur devis

$\square 505 € \quad \square$ Sur devis

$$
\begin{array}{cc}
\multicolumn{2}{c}{\text { Étudiants* }} \\
\text { Papier } & \text { Électronique } \\
+ & \text { seul } \\
\text { Électronique } & \text { seul }
\end{array}
$$

$\square 95 € \square \square 75 €$

$\square 135 € \quad \square 75 €$

\begin{tabular}{cc}
\multicolumn{2}{c}{ Enseignants* } \\
Papier & $\begin{array}{c}\text { Électronique } \\
+\end{array}$ \\
Électronique & seul \\
$\square 120 €$ & $\square 100 €$ \\
$\square 205 €$ & $\square 100 €$
\end{tabular}

